\title{
Prognostic role of nodal ratio, LODDS, pN in patients with pancreatic cancer with venous involvement
}

\author{
Giovanni Ramacciato', Giuseppe Nigri ${ }^{1 *}$ (D), Niccolo' Petrucciani ${ }^{1}$, Antonio Daniele Pinna ${ }^{2}$, Matteo Ravaioli², \\ Elio Jovine ${ }^{3}$, Francesco Minni ${ }^{4}$, Gian Luca Grazi ${ }^{5}$, Piero Chirletti ${ }^{6}$, Giuseppe Tisone ${ }^{7}$, Fabio Ferla ${ }^{8}$, \\ Niccolo' Napoli ${ }^{9}$ and Ugo Boggi ${ }^{9}$
}

\begin{abstract}
Background: The UICC/AJCC TNM staging system classifies lymph nodes as N0 and N1 in pancreatic cancer. Aim of the study is to determine whether the number of examine nodes, the nodal ratio (NR) and the logarithm odds of positive lymph nodes (LODDS) may better stratify the prognosis of patients undergoing pancreatectomy combined with venous resection for pancreatic cancer with venous involvement.

Methods: A multicenter database of 303 patients undergoing pancreatectomy in 9 Italian referral centers was analyzed. The prognostic impact of number of retrieved and examined nodes, NR, LODDS was analyzed and compared with ROC curves analysis, Pearson test, univariate and multivariate analysis.

Results: The number of metastatic nodes, pN, the NR and LODDS was significantly correlated with survival at multivariate analyses. The corresponding AUC for the number of metastatic nodes, pN, the NR and LODDS were $0.66,0.69,0.63$ and 0.65 , respectively. The Pearson test showed a significant correlation between the number of retrieved lymph nodes and number of metastatic nodes, $\mathrm{pN}$ and the NR. LODDS had the lower coefficient correlation. Concerning N1 patients, the NR, the LODDS and the number of metastatic nodes were able to significantly further stratify survival ( $p=0.040 ; p=0.046 ; p=0.038$, respectively).
\end{abstract}

Conclusions: The number of examined lymph nodes, the NR and LODDS are useful for further prognostic stratification of $\mathrm{N} 1$ patients in the setting of pancreatectomy combined with PV/SMV resection. No superiority of one over the others methods was detected.

Keywords: Pancreatic cancer, Tnm, Nodal ratio, Lodds, Prognosis, Nodal staging, Venous invasion, Portal vein, Superior mesenteric vein, Pancreatectomy

\section{Background}

Pancreatic cancer represents the fourth-leading cause of cancer-related mortality in the United States with an estimated 53,670 new cases in 2017 and 43,090 deaths [1]. In Europe, an estimated 103,773 new cases were reported in 2012 [2]. Lymph nodal status is an important prognostic factor in these patients, as a determinant for the appropriate prognostic stratification and therapeutic

\footnotetext{
* Correspondence: giuseppe.nigri@uniroma1.it; http://w3.uniroma1.it/nigri 'Department of Medical and Surgical Sciences and Translational Medicine, Faculty of Medicine and Psychology, St Andrea Hospital, Sapienza University, General Surgery Unit, Via di Grottarossa 1037, 00189 Rome, Italy

Full list of author information is available at the end of the article
}

decision-making [3]. Patients with pancreatic carcinoma with portal vein (PV) and/or superior mesenteric vein (SMV) invasion represent a particular challenge regarding prognostic analysis and treatment. The seventh edition of the International Union against Cancer (UICC) and the American Joint Committee on Cancer (AJCC) Tumor Node Metastasis (TNM) staging system classify regional lymph nodes as $\mathrm{NO}$ and N1, according to the presence of none or one or more nodal metastases [4]. The number of lymph nodes should be reported because it represents a prognostic factor, and N0 patients have a better prognosis with an increasing number of examined lymph nodes [5-8]. For optimal staging, the analysis of 
11-17 lymph nodes is recommended [5-9]. However, extended lymphadenectomy does not provide a survival advantage, according to randomized trials and meta-analyses [10-13]. In light of these data, the International Study Group of Pancreatic Surgery (ISGPS) agreed on a definition of standard lymphadenectomy [14]. Inaccurate surgical dissection, pathological evaluation or both may cause understaging for the suboptimal number of analyzed nodes, and subsequent inappropriate prognostic evaluation and error in clinical decisions [15].

To optimize nodal staging in patients with pancreatic cancer, different systems have been proposed and studied. The nodal ratio (NR) (ratio between metastatic and retrieved nodes) permits a subclassification of $\mathrm{N} 1$ patients, but it does not provide more information than TNM for NO patients. Several authors have shown that the NR is a significant prognostic factor for overall survival [16, 17]. LODDS (log odds of positive lymph nodes), defined as the logarithm of the ratio between the number of positive nodes and number of negative nodes, has thus been proposed as more effective than the NR in N0 patients [15].

Until now, few studies have compared all nodal staging systems in patients with pancreatic carcinoma and no data have been published on patients with PV/SMV venous invasion. Therefore, our aim is to analyze and compare different nodal staging systems in a subgroup of patients who underwent pancreatectomy with combined venous resection in nine Italian referral centers in order to identify the more advantageous nodal classification in this subset of patients.

\section{Methods}

The study included 303 patients who underwent pancreatectomy combined with PV or SMV resection for pancreatic carcinoma. The procedures were performed at nine Italian referral institutions. Some data retrieved from this multicenter database have already been published [18]. Written informed consent for participation in the study was obtained from participants.

\section{Preoperative work-up and surgical treatment}

The diagnosis of pancreatic adenocarcinoma was confirmed by pathological examination in all cases. Regarding neoadjuvant treatment, the multidisciplinary board of each unit established the indication and protocol, after imaging discussion among radiologists and clinicians.

Pancreaticoduodenectomy, left spleno-pancreatectomy or total pancreatectomy were performed according to the site of the lesion. Lymphadenectomy was performed as previously described in a standard fashion [14].

\section{Definition of clinical outcomes and pathological examination}

Postoperative mortality was defined as death during hospitalization or during the the first 30 days after pancreatectomy. For postoperative complications, ISGPS definitions were used [19, 20]. The presence of tumor cells within $1 \mathrm{~mm}$ from the margin was defined as R1 resection. Evidence of macroscopic residual tumor was defined as R2. ISGPS recommendations were followed [21].

\section{Lymph nodal staging systems}

$\mathrm{N}$ status was defined according to the AJCC staging system. The nodal ratio (NR) was defined as the ratio between the number of positive nodes and total harvested nodes. The classification system validated by Malleo et al. was chose for NR, after a careful review of the literature. Interval values were as follows: $0,0.01-0.2,0.21-$ 0.4 and more than 0.4 [3]. LODDS were calculated by $\log ($ pnod +0.5$) /($ tnod-pnod +0.5$)$, where pnod was the number of positive lymph nodes and tnod was the total number of examined nodes; 0.5 was added to both the numerator and the denominator to avoid an infinite number [15]. Patients were divided into two groups, dichotomizing the LODDS values around the median value. Each subgroup was further divided into two, dichotomizing again around the median LODDS value, resulting in four LODDS groups. The classification reported by Strobel et al. was chosen for the number of positive nodes, [8].

\section{Adjuvant therapies and follow-up}

The multidisciplinary tumor board of each institution validated the indication for adjuvant chemotherapy or radiochemotherapy. Decision was based on patients' performance status and pathological results. During followup, physical examination and CA 19-9 determination were scheduled every 3 months in the first 2 years and than every 6 months, and thoraco-abdominal CT scan every 6 months in the first 2 years.

\section{Statistical analysis}

The multicenter database was prospectively collected by each center and retrospectively analyzed. T-test for continuous variables and the chi-square test for categorical variables were used to calculate differences in distribution. Overall survival rates were calculated according to the Kaplan-Meier method, and we used the log-rank test to assess the statistical differences between different groups. Univariate and multivariate analyses were used to identify the most significant. Correlation between nodal staging systems and prognosis was assessed with univariate and multivariate analyses. Variables significant in the univariate analysis were used for the multivariate 
model, analyzing separately each nodal staging system. Overall survival rates were calculated according to different $\mathrm{pN}$, NR, LODDS, number of metastatic nodes and number of retrieved nodes. ROC curves analysis was used to evaluate the accuracy of the different nodal staging systems, using nodal classifications as variables and 5 -year survival as classification variables. The method of Delong et al. [22] was used. Pearson correlation coefficient was used to calculate the correlations between number of retrieved nodes, number of metastatic nodes, NR and LODDS. $p<0.05$ was considered as statistically significant. Statistical analyses were performed by using MedCalc for Windows, version 10.2.0.0 (MedCalc Software, Belgium).

\section{Results}

Patients' characteristics, preoperative work-up and treatment and surgery

The study population was composed of 165 men (54.5\%) and 138 women $(45.5 \%)$. The majority of patients were classed as N1 (70.6\%). Patients' characteristics according to nodal status are listed in Table 1 . One hundred and eighty-seven patients (61.7\%) had one or more comorbidity. Cardiovascular comorbidities were detected in $49.5 \%$ of patients, respiratory comorbidities in $12.2 \%$ and metabolic comorbidities in $29.0 \%$.

Mean tumor diameter according to the CT scan was $32.9 \pm 15.4 \mathrm{~mm}$. Preoperative biliary drainage was performed in $28.6 \%$ of cases, and neoadjuvant chemotherapy was administered to $6.4 \%$ of patients.

The majority of patients underwent pancreaticoduodenectomy (PD) (76.9\%), 60 (19.8\%) underwent left pancreatectomy (LP) and 10 (3.3\%) total pancreatectomy (TP) (Table 1). All patients underwent portal or superior mesenteric vein resection. Mean operative time was $462.6 \pm 134.2 \mathrm{~min}$ and blood loss $475.2 \pm 401.6 \mathrm{ml}$.

\section{Postoperative outcomes, pathological and lymph nodal analysis and survival}

Complications occurred in $49.8 \%$ of patients and mortality in $6.6 \%$ (Table 2). Postoperative pancreatic fistula occurred in $11.9 \%$ and delayed gastric emptying in $23.1 \%$. Mean intensive care unit stay was $3.2 \pm 4.6$ days. Mean hospital stay was $20.4 \pm 11.6$ days. Histological venous invasion was found in $53.8 \%$ of venous specimens. Mean tumor diameter was $35.0 \pm 20.7 \mathrm{~mm}$. The mean number of retrieved lymph nodes was $33.5 \pm 22.6$ and ranged from 2 to 131. The mean number of metastatic nodes was $3.4 \pm 4.5$, ranging from 0 to 25 . Patients undergoing LP had a significantly higher number of retrieved lymph nodes than patients undergoing PD $(47.9 \pm 25$ versus $29.6 \pm 20.2 ; p<0.0001)$. The mean number of metastatic nodes was not different in patients submitted to PD (3.1 $\pm 4)$, LP $(4.4 \pm 5.8)$ and TP $(3.7 \pm 4.9)$. The resection margin was tumor-free in $73.3 \%$ of cases. Adjuvant therapy was administered to $72.1 \%$ of patients. Mean follow-up duration was 37.9 months. Median overall survival was 25 months and five-year survival rate was $25.2 \%$.

\section{Analysis of prognostic factors}

Table 3 shows survival according to patient and tumor characteristics. Factors that significantly correlated with overall survival were the number of metastatic nodes, $\mathrm{pN}$, the NR and LODDS, whereas no correlation was

Table 1 Patients' characteristics and procedures. Data are presented for the entire cohort and according to nodal status. Continuous variables are presented as mean \pm SD

\begin{tabular}{|c|c|c|c|c|}
\hline Variable & No (89) & N1 (214) & Total (303) & $p$ \\
\hline Age & $65.7 \pm 10.7$ & $67.4 \pm 10.5$ & $66.9 \pm 10.6$ & 0.223 \\
\hline Sex, Females & $40.7 \%$ & $47.5 \%$ & $45.5 \%$ & 0.348 \\
\hline ASA score & $2.3 \pm 0.7$ & $2.5 \pm 0.7$ & $2.4 \pm 0.7$ & 0.379 \\
\hline $\mathrm{BMI}$ & $23.8 \pm 2.9$ & $24.1 \pm 3.2$ & $24.0 \pm 3.1$ & 0.651 \\
\hline Comorbidities & $57.0 \%$ & $63.5 \%$ & $61.7 \%$ & 0.381 \\
\hline CEA (Ul/ml) & $15.7 \pm 33.7$ & $10.7 \pm 19.1$ & $12.0 \pm 23.6$ & 0.452 \\
\hline CA 19.9 (Ul/ml) & $592 \pm 1276$ & $644 \pm 1375$ & $637.0 \pm 1345$ & 0.831 \\
\hline Albumin (g/dl) & $3.8 \pm 0.6$ & $3.7 \pm 0.6$ & $3.7 \pm 0.6$ & 0.258 \\
\hline Bilirubin (mg/dl) & $6.7 \pm 15.7$ & $6.2 \pm 6.3$ & $6.3 \pm 9.7$ & 0.745 \\
\hline Tumor diameter at $\mathrm{CT}, \mathrm{mm}$ & $36.3 \pm 22.6$ & $31.6 \pm 11.7$ & $32.9 \pm 15.4$ & 0.103 \\
\hline Surgery & & & & 0.747 \\
\hline PD, number & 68 & 165 & 233 & \\
\hline$L P$, number & 18 & 42 & 60 & \\
\hline TP, number & 3 & 7 & 10 & \\
\hline
\end{tabular}

SD standard deviation, ASA American Society of Anesthesiology, BMI Body Mass Index, CEA carcinoembryonic antigen, CA 19.9 Carbohydrate Antigen 19.9, CT computed tomography, $P D$ pancreaticoduodenectomy, $L P$ left pancreatectomy, $T P$ total pancreatectomy 
Table 2 Postoperative complications and mortality in 303 patients submitted to pancreatectomy with portal vein and/or superior mesenteric vein resection

\begin{tabular}{|c|c|c|}
\hline Variable & $\mathrm{N}$. & $\%$ \\
\hline Overall complications & 151 & $49.8 \%$ \\
\hline Mortality & 20 & $6.6 \%$ \\
\hline Pancreatic fistula & 36 & $11.9 \%$ \\
\hline Grade A & 16 & \\
\hline Grade B & 14 & \\
\hline Grade C & 6 & \\
\hline DGE & 70 & $23.1 \%$ \\
\hline Grade A & 32 & \\
\hline Grade B & 25 & \\
\hline Grade C & 13 & \\
\hline Non pancreatic leak & 9 & $3.0 \%$ \\
\hline Postoperative bleeding & 18 & $5.9 \%$ \\
\hline Re-laparotomy & 21 & $6.9 \%$ \\
\hline PV-SMV thrombosis & 5 & $1.7 \%$ \\
\hline Abdominal collection & 33 & $10.9 \%$ \\
\hline $\begin{array}{l}\text { Need of postoperative abdominal } \\
\text { drain }\end{array}$ & 36 & $11.9 \%$ \\
\hline Wound infection & 16 & $5.3 \%$ \\
\hline Urinary tract infection & 3 & $1.0 \%$ \\
\hline Cardiovascular complications & 6 & $2.0 \%$ \\
\hline DVT/PE & 5 & $1.6 \%$ \\
\hline Acute renal failure & 3 & $1.0 \%$ \\
\hline Pneumonia & 4 & $1.3 \%$ \\
\hline
\end{tabular}

found for age, sex, comorbidities, tumor size, number of retrieved nodes, $\mathrm{T}$ stage and resection margin. Figs. 1, 2, 3 and 4 show the survival curves according to the number of metastatic nodes, $\mathrm{pN}$, the $\mathrm{NR}$ and LODDS, respectively. According to the multivariate analyses, the number of metastatic nodes, $\mathrm{pN}$, the NR and LODDS were significantly correlated with survival (Table 4).

\section{Comparison between $\mathrm{pN}$ staging, the NR and LODDS methods}

The corresponding AUC for the number of metastatic nodes, pN, the NR and LODDS were 0.66 (95\% CI 0.58 to 0.73 ), 0.69 (95\% CI 0.62 to 0.76 ), 0.63 (95\% CI 0.55 to 0.70 ) and 0.65 (95\% CI 0.57 to 0.72 ), respectively, with no significant differences (Fig. 5). The scatter plot of the relationship between LODDS and the NR is reported in Fig. 6. The LODDS value increased with the ratio of metastatic lymph nodes, showing correlation between LODDS and the NR. Values of LODDS were still heterogeneous, even in cases with $\mathrm{NR}=0$. A significant
Table 3 Clinicopathological data and univariate survival analysis results of 303 patients submitted to pancreatectomy with portal vein and/or superior mesenteric vein resection

\begin{tabular}{|c|c|c|c|}
\hline Variable & Patients (\%) & $\begin{array}{l}\text { Median survival } \\
\text { (months) }\end{array}$ & $\begin{array}{l}p \text { (univariate } \\
\text { analysis) }\end{array}$ \\
\hline Age & & & 0.531 \\
\hline$<70$ & 53.2 & 28.3 & \\
\hline$\geq 70$ & 46.8 & 24 & \\
\hline Sex & & & 0.094 \\
\hline Males & 54.5 & 25 & \\
\hline Females & 45.5 & 26 & \\
\hline Comorbidities & & & 0.058 \\
\hline No & 38.3 & 26 & \\
\hline Yes & 61.7 & 24 & \\
\hline Tumor size & & & 0.193 \\
\hline$<30 \mathrm{~mm}$ & 37.3 & 28 & \\
\hline$\geq 30 \mathrm{~mm}$ & 62.7 & 24 & \\
\hline Resection margin & & & 0.850 \\
\hline Ro & 73.3 & 27 & \\
\hline $\mathrm{R} 1$ & 26.7 & 23 & \\
\hline T stage & & & 0.506 \\
\hline 1 & 0.7 & na & \\
\hline 2 & 6.6 & 28 & \\
\hline 3 & 86.1 & 24 & \\
\hline 4 & 6.6 & 22 & \\
\hline $\begin{array}{l}\text { Number of retrieved } \\
\text { lymph nodes }\end{array}$ & & & 0.797 \\
\hline$<17$ & 24.1 & 26 & \\
\hline$\geq 17$ & 75.9 & 24 & \\
\hline $\begin{array}{l}\text { Number of metastatic } \\
\text { lymph nodes }\end{array}$ & & & 0.0005 \\
\hline $0-2$ & 56.1 & 35 & \\
\hline$\geq 3$ & 43.9 & 22 & \\
\hline$N$ stage & 0.0002 & & \\
\hline No & 29.4 & 46 & \\
\hline N1 & 70.6 & 23 & \\
\hline NR & 0.0005 & & \\
\hline 0 & 29.4 & 43 & \\
\hline $0.01-0.2$ & 48.2 & 24 & \\
\hline $0.21-0.4$ & 16.2 & 17 & \\
\hline$>0.4$ & 6.3 & 22 & \\
\hline LODDS & & & 0.0013 \\
\hline Lodds $<-0.005$ & 23.4 & 72 & \\
\hline$-0.005 \leq$ Lodds $<0.012$ & 24.8 & 32 & \\
\hline $0.012 \leq$ Lodds $<0.026$ & 26.1 & 22 & \\
\hline Lodds $\geq 0.026$ & 25.7 & 22 & \\
\hline
\end{tabular}

NR nodal ratio, LODDS log odds of positive lymph nodes

correlation between the number of retrieved lymph nodes and number of metastatic nodes was found at Pearson test (Table 5). The correlation values were lower for $\mathrm{pN}$ and the NR, and LODDS had the lower 


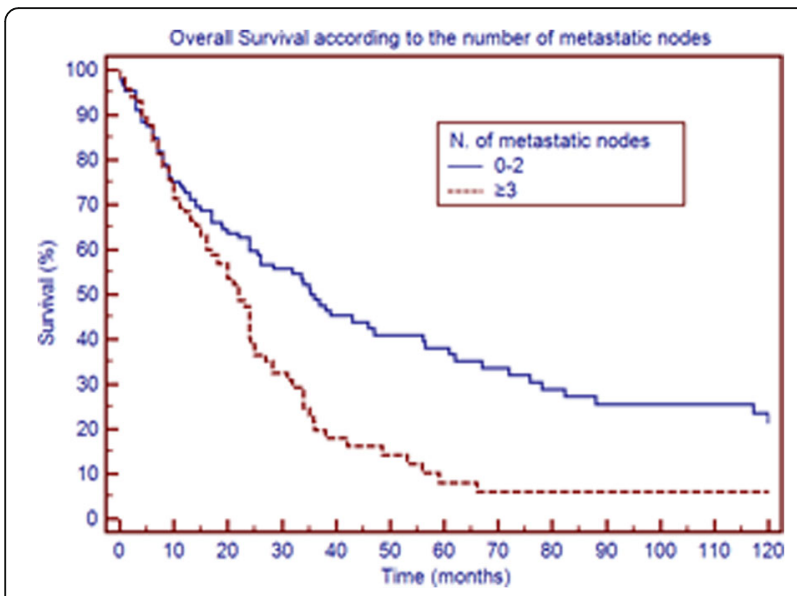

Fig. 1 Overall survival according to the number of metastatic nodes

coefficient correlation (0.079) with the number of retrieved nodes $(p=0.154)$.

\section{Further stratification of N1 and N0 patients}

Concerning N1 patients, the NR was able to further stratify survival, with patients with $\mathrm{NR}<0.2$ having better prognosis (median survival of 24 versus 19 months, $p=0.040)$. Further, LODDS stratified N1 patients into two groups with different prognoses, with patients of LODDS >0.03 having significantly worse survival (20 versus 24 months, $p=0.046$ ). In addition, the number of metastatic nodes was able to stratify N1 patients in this series, according to the classification reported by Strobel et al. $(p=0.038)$. In the group of patients with $<17$ retrieved lymph nodes, $\mathrm{pN}$, the NR and LODDS were all able to stratify patient survival $(p=0.01, p=0.023$ and $p=0.05$, respectively). The LODDS classification was used to stratify the 89 N0 patients; LODDS1 patients had a median survival of 72 months, whereas

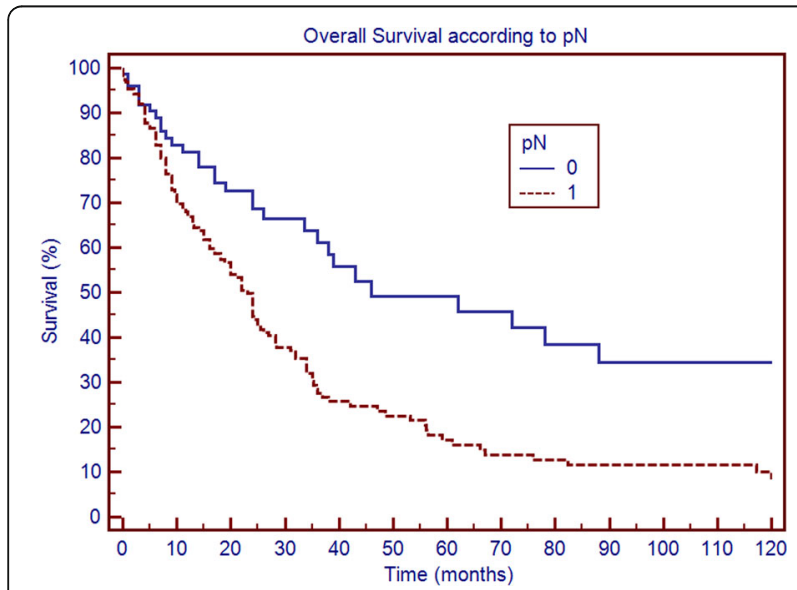

Fig. 2 Overall survival according to $\mathrm{pN}$ status



Fig. 3 Overall survival according to the NR

LODDS2 patients had a median survival of 36 months (not statistically significant; $p=0.229$ ).

\section{Discussion}

Lymph nodal status is considered to be one of the most important prognostic factors after pancreatectomy for adenocarcinoma. The most used nodal staging system is the $\mathrm{N}$ status of the AJCC classification, which identifies N0 and N1 patients, according to the presence or absence of nodal metastases. Previous studies have analyzed the prognostic role of the number of examined lymph nodes, number of pathologic lymph nodes, the NR and LODDS in patients with pancreatic cancer, with different results $[5,6,8,23,24]$. The number of positive nodes has been suggested to stratify N1 patients, adding prognostic information $[8,25]$. Strobel and colleagues reported a median survival of 31.1, 26.1, 21.9 and 18.3 months in patients with $1,2-3,4-7$ and $>7$ positive nodes, respectively [8]. The role of the number of positive nodes was also shown in patients submitted to

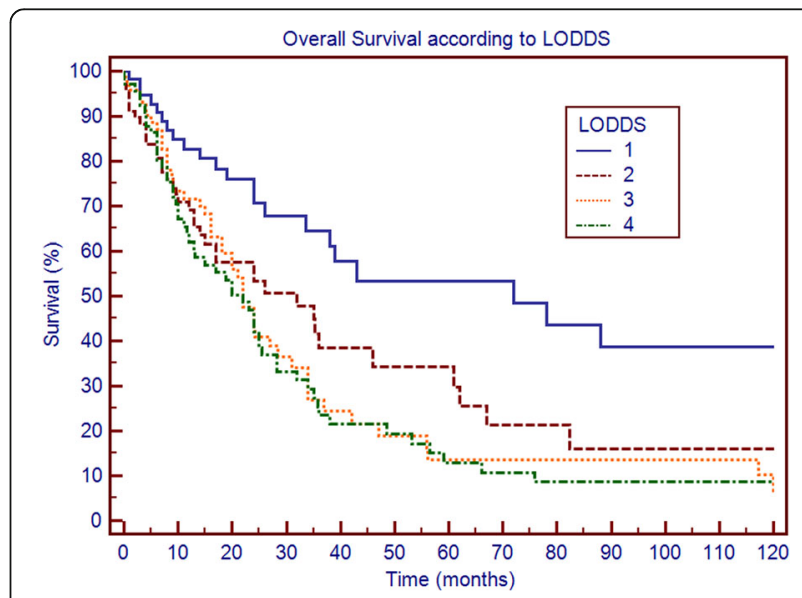

Fig. 4 Overall survival according to LODDS 
Table 4 Multivariate analyses using the Cox proportional hazard method. Evaluation of prognostic impact of number of metastatic nodes, pN, nodal ratio and LODDS

\begin{tabular}{|c|c|c|c|c|c|}
\hline Variable & $b$ & SE & $P$ & $\operatorname{Exp}(b)$ & $95 \% \mathrm{Cl}$ \\
\hline Age & -0.100 & 0.184 & 0.585 & 0.905 & 0.633 to 1.294 \\
\hline Sex & -0.244 & 0.188 & 0.196 & 0.784 & 0.543 to 1.132 \\
\hline Tumor size & 0.176 & 0.197 & 0.372 & 1.192 & 0.813 to 1.749 \\
\hline R status & -0.089 & 0.221 & 0.686 & 0.915 & 0.594 to 1.407 \\
\hline T stage & -0.059 & 0.193 & 0.759 & 0.942 & 0.646 to 1.374 \\
\hline N. met. Nodes & 0.427 & 0.186 & 0.022 & 1.533 & 1.066 to 2.23 \\
\hline Age & -0.013 & 0.183 & 0.945 & 0.987 & 0.691 to 1.411 \\
\hline Sex & -0.247 & 0.189 & 0.192 & 0.781 & 0.540 to 1.130 \\
\hline Tumor size & 0.190 & 0.197 & 0.336 & 1.209 & 0.823 to 1.775 \\
\hline R status & -0.085 & 0.218 & 0.698 & 0.919 & 0.600 to 1.407 \\
\hline T stage & -0.115 & 0.195 & 0.555 & 0.892 & 0.610 to 1.303 \\
\hline stage & 0.603 & 0.239 & 0 & 1.828 & 911 \\
\hline Age & -0.0812 & 0.183 & 0.655 & 0.922 & 0.646 to 1.316 \\
\hline Sex & -0.195 & 0.190 & 0.305 & 0.823 & 0.568 to 1.192 \\
\hline Tumor size & 0.206 & 0.196 & 0.293 & 1.229 & 0.838 to 1.803 \\
\hline R status & -0.170 & 0.221 & 0.441 & 0.843 & 0.548 to 1.298 \\
\hline T stage & -0.019 & 0.188 & 0.920 & 0.981 & 0.680 to 1.416 \\
\hline Nodal ratio & 0.384 & 0.108 & 0.001 & 1.468 & 1.189 to 1.811 \\
\hline ge & -0.010 & 0.183 & 0.955 & 0.989 & 0.692 to 1.415 \\
\hline Sex & -0.229 & 0.188 & 0.221 & 0.795 & 0.551 to 1.146 \\
\hline Tumor size & 0.184 & 0.197 & 0.349 & 1.202 & 0.819 to 1.764 \\
\hline R status & -0.189 & 0.222 & 0.395 & 0.828 & 0.537 to 1.277 \\
\hline T stage & -0.114 & 0.197 & 0.563 & 0.892 & 0.608 to 1.310 \\
\hline LODDS & 0.273 & 0.089 & 0.002 & 1.313 & 1.105 to 1.562 \\
\hline
\end{tabular}

N. met. Nodes number of metastatic nodes, NR nodal ratio, LODDS log odds of positive lymph nodes

pancreatic surgery after neoadjuvant therapy [25]. Concerning the NR, a number of authors have demonstrated its ability to further stratify node-positive patients [26, 27].

LODDS are new prognostic parameters, which aim to better stratify patients regarding their nodal metastases status. In the setting of gastric, colorectal, breast and other neoplasms, promising data have been reported [28-30]. Comparing to NR, which is a function of the number of retrieved nodes, LODDS is a function of the number of negative lymph nodes. In the setting of pancreatic cancer, only one study has analyzed this parameter, suggesting the advantage of LODDS over the NR in node-negative patients [15]. Patients with pancreatic cancer and portal vein/superior mesenteric vein axes involvement represent a peculiar and challenging subset of patients. Several questions are still open in this setting regarding better perioperative treatment, surgical strategies and prognostic stratification. No study has thus far

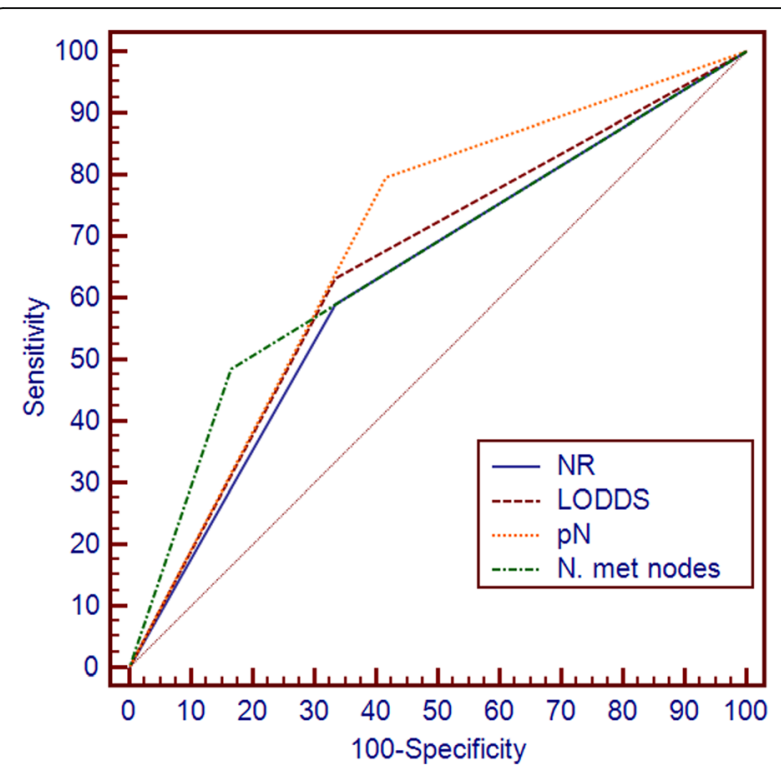

Fig. 5 Comparison of ROC curves analysis between the number of metastatic nodes, pN status, the NR and LODDS

analyzed the nodal staging system in this subset of patients to our knowledge, and for these reasons we reviewed a multicenter database to report our data about nodal prognostic factors in patients with venous invasion.

Our study analyzed a population of 303 patients undergoing pancreatectomy combined with venous resection. Patients were treated in referral centers for pancreatic pathology, and standard lymphadenectomy, as recommended by the ISGPS, was performed. The mean number of retrieved lymph nodes was high (33.5), and the majority of patients $(70.6 \%)$ had at least one metastatic node. Patients submitted to LP had a higher number of retrieved nodes, whereas the number of metastatic nodes was not different in patients

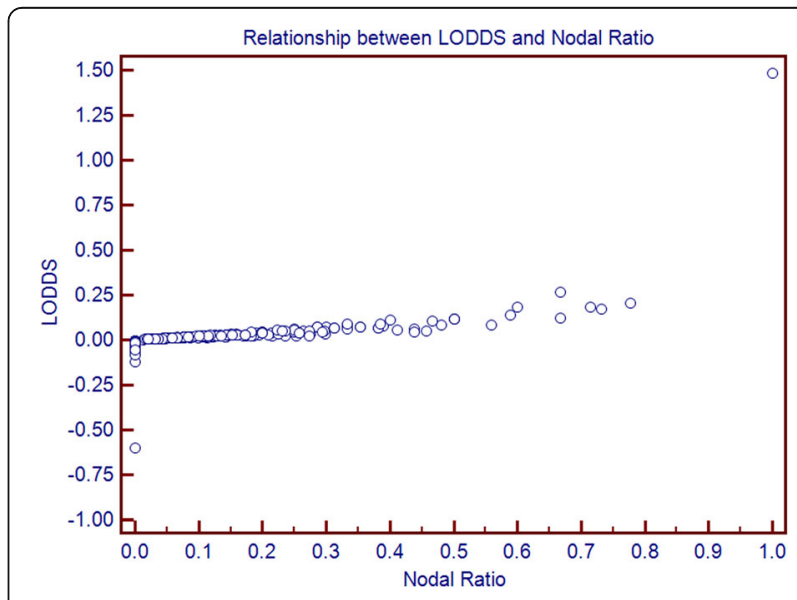

Fig. 6 Relationship between LODDS and the NR 
Table 5 Pearson correlation test between number of retrieved lymph nodes and number of metastatic nodes, $\mathrm{N}$ status, NR, LODDS

\begin{tabular}{ll} 
Correlation between of retrieved lymph nodes and number or \\
metastatic nodes \\
Sample size & 303 \\
Correlation coefficient $r$ & 0.298 \\
Significance level & $p<0.0001$ \\
$95 \%$ coefficient interval for $r$ & 0.192 to 0.398 \\
Correlation between of retrieved lymph nodes and $\mathrm{N}$ status \\
Sample size & 303 \\
Correlation coefficient $r$ & 0.1276 \\
Significance level & $p=0.026$ \\
95\% coefficient interval for $r$ & 0.015 to 0.237 \\
Correlation between of retrieved lymph nodes and $\mathrm{N}$ ratio \\
Sample size & 303 \\
Correlation coefficient $r$ & -0.193 \\
Significance level & $p=0.001$ \\
$95 \%$ coefficient interval for $r$ & -0.299 to -0.082 \\
Correlation between of retrieved lymph nodes and LODDS \\
Sample size & 303 \\
Correlation coefficient $r$ & -0.082 \\
Significance level & $p=0.154$ \\
$95 \%$ coefficient interval for $r$ & -0.193 to 0.031 \\
\hline &
\end{tabular}

NR nodal ratio, LODDS log odds of positive lymph nodes

undergoing PD, LP or TP. Univariate and multivariate analyses of prognostic factors were performed. Nodal staging indexes were significant predictors of survival, and the multivariate analysis confirmed the significant prognostic value of the number of metastatic nodes, $\mathrm{pN}$, the NR, and LODDS. A comparison of the different systems was attempted to demonstrate the superiority of one of them. The ROC curves' comparison did not show any significant differences. LODDS had a lower and non-significant correlation with the number of retrieved nodes according to the Pearson test, which may be advantageous in the case of inadequate lymphadenectomy (in this series, only $12.5 \%$ of patients has fewer than 11 retrieved nodes). A scatter plot was presented to show that LODDS has the power to discriminate patients with the same NR $(0$ or 1) but a different prognosis. However, in the entire cohort, all nodal staging systems seemed to be efficacious with a strong prognostic significance.

We further studied the group of patients having at least one nodal metastasis. Clearly, $\mathrm{pN}$ classification is limited in this setting, because all patients are classified as N1. The comparison of survival curves via the logrank test demonstrated that the NR, the number of positive nodes and LODDS might all provide further stratification for these patients. This result is concordant with those of previous studies, and confirms that $\mathrm{pN}$ staging may also be integrated by further information. The number of positive nodes is easy to retrieve and does not require calculation. However, patients with different prognosis may have the same values. For example, the number of positive nodes is the same for patients having 4 metastatic nodes out of 4 retrieved ( $100 \%$ of metastatic nodes) or 4 out of $40(10 \%)$, for example. The NR is simple to calculate. NR carries information that are related to both the number of metastatic and retrieved nodes. However, for values approaching 1 its accuracy seems to diminish (no difference between a patient with 1/1 metastasis and one with 40/40). Furthermore, further stratification of N0 patients is not possible using NR and number of positive nodes. LODDS represent a nodal prognostic index, which is more complex to understand. Furthermore, calculation is less simple, which explain why is rarely used in clinical practice. Theoretically, LODDS have several advantages, including the possibility to further stratify N0 patients. In our series, we failed to demonstrate a statistically significant difference in the 89 N0 patients using LODDS, but these results were limited by the sample size of NO patients in our study population.

The novelty of this study is that it is the first one evaluating different nodal staging systems in the setting of patients undergoing pancreatectomy with synchronous venous resection. Patients with portal vein or superior mesenteric vein invasion represent a challenging subset of patients, and optimal prognostic stratification is needed in their clinical management. We demonstrated that N1 patients might be further classified using the number of examined lymph nodes, the NR and LODDS. Furthermore, our study adds useful information on the role of LODDS and pancreatic cancer, which is still controversial. Only a few studies have been published about LODDS in pancreatic cancer staging, with some authors suggesting its utility [15] and others recommending avoiding its use [31].

We point out some limitations of this study. Data regarding disease-free survival were not analyzed, because not all included centres reported the information. Furthermore, the study is retrospective. However, use of ISGPS definition and the numbers of included patients represent some remarkable aspects of this series. In this study, neoadjuvant therapy was administered only to a minority of patients. We can explain this data analyzing NCCN guidelines until 2014. Up-front surgery was indicated in fit patients with venous invasion at CT scan suitable to resection and reconstruction with complete tumor clearance. Furthermore, neoadjuvant therapy is a factor that may modify nodal status; hence, the low rate of neoadjuvant therapy in this study represents an 
advantage regarding the analysis of nodal prognostic factors.

\section{Conclusions}

In conclusion, in patients undergoing pancreatectomy with combined PV/SMV resection for pancreatic cancer, the number of examined lymph nodes, the NR and LODDS are useful for the further prognostic stratification of N1 patients. All these staging systems permit the better stratification of patients with nodal metastases and are useful in clinical practice. No superiority of one over the others was detected in patients undergoing pancreatectomy with venous resection.

\section{Acknowledgements}

Not applicable

\section{Funding}

No founding was received.

\section{Availability of data and materials}

The datasets used and/or analyzed during the current study are available from the corresponding author on reasonable request.

\section{Authors' contributions \\ GR, GN, NP, have made substantial contributions to conception and design, acquisition of data, analysis and interpretation of data; 2) have been involved in drafting the manuscript and revising it critically for important intellectual content; 3) have given final approval of the version to be published; and 4) agree to be accountable for all aspects of the work in ensuring that questions related to the accuracy or integrity of any part of the work are appropriately investigated and resolved. ADP, MR, EJ, FM, GLG, PC, GT, FF, NN, UB have made substantial contributions to acquisition of data, analysis and interpretation of data; 2) have been involved in revising the manuscript critically for important intellectual content; 3) have given final approval of the version to be published; and 4) agree to be accountable for all aspects of the work in ensuring that questions related to the accuracy or integrity of any part of the work are appropriately investigated and resolved.}

\section{Ethics approval and consent to participate}

Ethics approval was obtained by the Ethics Committee of Sapienza University of Rome.

Written informed consent for participation in the study was obtained from participants.

Research was in compliance with the Declaration of Helsinki (http:// www.wma.net/en/30publications/10policies/b3/index.html).

\section{Consent for publication}

Not applicable

\section{Competing interests}

The authors declare that they have no competing interests. Prof. Giuseppe Nigri is a member of the board (Associate Editor) of BMC Series journal.

\section{Publisher's Note}

Springer Nature remains neutral with regard to jurisdictional claims in published maps and institutional affiliations.

\section{Author details}

'Department of Medical and Surgical Sciences and Translational Medicine, Faculty of Medicine and Psychology, St Andrea Hospital, Sapienza University, General Surgery Unit, Via di Grottarossa 1037, 00189 Rome, Italy. 2Department of Medical and Surgical Sciences-DIMEC, S. Orsola-Malpighi Hospital, Alma Mater Studiorum, University of Bologna, General Surgery and Transplantation Unit, Bologna, Italy. ${ }^{3}$ General Surgery Unit, 'Maggiore' Hospital, Bologna, Italy. ${ }^{4}$ Department of Medical and Surgical Sciences (DIMEC), Alma Mater Studiorum, S. Orsola-Malpighi Hospital, University of
Bologna, General Surgery Unit, Bologna, Italy. ${ }^{5}$ Regina Elena National Cancer Institute IFO, Hepato-pancreato-biliary Surgery Unit, Rome, Italy. ${ }^{6}$ Department of Surgical Sciences, Sapienza University of Rome, Policlinico Umberto I Hospital, General Surgery Unit, Rome, Italy. ${ }^{7}$ Department of Experimental Medicine and Surgery, Liver Unit, Tor Vergata University of Rome, Rome, Italy. ${ }^{8}$ Division of General Surgery and Transplantation Surgery, Niguarda Hospital, Milan, Italy. ${ }^{9}$ Division of General Surgery and Transplantation Surgery, Pisa University Hospital, Pisa, Italy.

Received: 4 September 2017 Accepted: 15 November 2017

Published online: 23 November 2017

\section{References}

1. Siegel RL, Miller KD, Jemal A. Cancer statistics, 2017. CA Cancer J Clin. 2017; 67:7-30.

2. New European Cancer Observatory - IARC; Cancer factsheets. Available at: http://eco.iarc.fr/eucan/Cancer.aspx?Cancer=15. Accessed 13 Jul 2015.

3. Malleo G, Maggino L, Capelli P, et al. Reappraisal of nodal staging and study of lymph node station involvement in pancreaticoduodenectomy with the standard international study group of pancreatic surgery definition of lymphadenectomy for cancer. J Am Coll Surg. 2015;221:367-79.

4. Pancreas Cancer Staging, American Joint Committee on Cancer, $7^{\text {th }}$ Edition. Available at: https://cancerstaging.org/references-tools/quickreferences/ Documents/PancreasSmall.pdf. Accessed 1 Apr 2016.

5. Valsangkar NP, Bush DM, Michaelson JS, et al. NO/N1, PNL, or LNR? The effect of lymph node number on accurate survival prediction in pancreatic ductal adenocarcinoma. J Gastrointest Surg. 2013;17:257-66.

6. Huebner M, Kendrick M, Reid-Lombardo KM, et al. Number of lymph nodes evaluated: prognostic value in pancreatic adenocarcinoma. J Gastrointest Surg. 2012;16:920-6

7. Opfermann KJ, Wahlquist AE, Garrett-Mayer E, Shridhar R, Cannick L, Marshall DT. Adjuvant radiotherapy and lymph node status for pancreatic cancer: results of a study from the surveillance, epidemiology, and end results (SEER) registry data. Am J Clin Oncol. 2014;37:112-6.

8. Strobel $\mathrm{O}$, Hinz U, Gluth $\mathrm{A}$, et al. Pancreatic adenocarcinoma: number of positive nodes allows to distinguish several N categories. Ann Surg. 2015;261:961-9.

9. Ashfaq A, Pockaj BA, Gray RJ, Halfdanarson TR, Wasif N. Nodal counts and lymph node ratio impact survival after distal pancreatectomy for pancreatic adenocarcinoma. J Gastrointest Surg. 2014;18:1929-35.

10. Michalski CW, Kleeff J, Wente MN, Diener MK, Büchler MW, Friess $H$. Systematic review and meta-analysis of standard and extended lymphadenectomy in pancreaticoduodenectomy for pancreatic cancer. $\mathrm{Br} J$ Surg. 2007:94:265-73.

11. Sun J, Yang $Y$, Wang $X$, et al. Meta-analysis of the efficacies of extended and standard pancreatoduodenectomy for ductal adenocarcinoma of the head of the pancreas. World J Surg. 2014;38:2708-15.

12. Jang JY, Kang MJ, Heo JS, et al. A prospective randomized controlled study comparing outcomes of standard resection and extended resection, including dissection of the nerve plexus and various lymph nodes, in patients with pancreatic head cancer. Ann Surg. 2014;259: 656-64.

13. Dasari BV, Pasquali S, Vohra RS, et al. Extended versus standard lymphadenectomy for pancreatic head cancer: meta-analysis of randomized controlled trials. J Gastrointest Surg. 2015;19:1725-32.

14. Tol JA, Gouma DJ, Bassi C, et al. Definition of a standard lymphadenectomy in surgery for pancreatic ductal adenocarcinoma: a consensus statement by the international study group on pancreatic surgery (ISGPS). Surgery. 2014;156:591-600

15. La Torre M, Nigri G, Petrucciani N, et al. Prognostic assessment of different lymph node staging methods for pancreatic cancer with $\mathrm{RO}$ resection: $\mathrm{pN}$ staging, lymph node ratio, log odds of positive lymph nodes. Pancreatology. 2014;14:289-94.

16. Liu ZQ, Xiao ZW, Luo GP, et al. Effect of the number of positive lymph nodes and lymph node ratio on prognosis of patients after resection of pancreatic adenocarcinoma. Hepatobiliary Pancreat Dis Int. 2014;13:634-41.

17. Slidell MB, Chang DC, Cameron JL, et al. Impact of total lymph node count and lymph node ratio on staging and survival after pancreatectomy for pancreatic adenocarcinoma: a large, population-based analysis. Ann Surg Oncol. 2008;15:165-74 
18. Ramacciato G, Nigri G, Petrucciani N, et al. Pancreatectomy with mesenteric and portal vein resection for borderline resectable pancreatic cancer: multicenter study of 406 patients. Ann Surg Oncol. 2016; [Epub ahead of print]

19. Bassi C, Dervenis C, Butturini $G$, et al. Postoperative pancreatic fistula: an international study group (ISGPF) definition. Surgery. 2005;138:8-13.

20. Wente MN, Bassi C, Dervenis C, et al. Delayed gastric emptying (DGE) after pancreatic surgery: a suggested definition by the international study Group of Pancreatic Surgery (ISGPS). Surgery. 2007;142:761-8.

21. Bockhorn M, Uzunoglu FG, Adham M, et al. Borderline resectable pancreatic cancer: a consensus statement by the international study Group of Pancreatic Surgery (ISGPS). Surgery. 2014;155:977-88.

22. DeLong ER, DeLong DM, Clarke-Pearson DL. Comparing the areas under two or more correlated receiver operating characteristic curves: a nonparametric approach. Biometrics. 1988;44:837-45.

23. Kang MJ, Jang JY, Chang YR, Kwon W, Jung W, Kim SW. Revisiting the concept of lymph node metastases of pancreatic head cancer: number of metastatic lymph nodes and lymph node ratio according to $\mathrm{N}$ stage. Ann Surg Oncol. 2014;21:1545-51.

24. Riediger $\mathrm{H}$, Keck $\mathrm{T}$, Wellner $\mathrm{U}$, et al. The lymph node ratio is the strongest prognostic factor after resection of pancreatic cancer. J Gastrointest Surg. 2009;13:1337-44.

25. Fischer $L K$, Katz $M H$, Lee $S M$, et al. The number and ratio of positive lymph nodes affect pancreatic cancer patient survival after neoadjuvant therapy and pancreaticoduodenectomy. Histopathology. 2016;68:210-20.

26. Yamada S, Fujii T, Hirakawa A, Kanda M, Sugimoto H, Kodera Y. Lymph node ratio as parameter of regional lymph node involvement in pancreatic cancer. Langenbeck's Arch Surg. 2016; [Epub ahead of print]

27. Robinson SM, Rahman A, Haugk B, et al. Metastatic lymph node ratio as an important prognostic factor in pancreatic ductal adenocarcinoma. Eur J Surg Oncol. 2012;38:333-9.

28. Wen J, Ye F, He X, et al. Development and validation of a prognostic nomogram based on the log odds of positive lymph nodes (LODDS) for breast cancer. Oncotarget. 2016;

29. Song YX, Gao P, Wang ZN, et al. Which is the most suitable classification for colorectal cancer, log odds, the number or the ratio of positive lymph nodes? PLoS One. 2011;6:e28937.

30. Aurello P, Petrucciani N, Nigri GR, et al. Log odds of positive lymph nodes (LODDS): what are their role in the prognostic assessment of gastric adenocarcinoma? J Gastrointest Surg. 2014;18:1254-60.

31. Riediger H, Kulemann B, Wittel U, et al. Prognostic role of log odds of lymph nodes after resection of pancreatic head cancer. J Gastrointest Surg. 2016:20:1707-15.

\section{Submit your next manuscript to BioMed Central and we will help you at every step:}

- We accept pre-submission inquiries

- Our selector tool helps you to find the most relevant journal

- We provide round the clock customer support

- Convenient online submission

- Thorough peer review

- Inclusion in PubMed and all major indexing services

- Maximum visibility for your research

Submit your manuscript at www.biomedcentral.com/submit

C Biomed Central 